\title{
Tribute to the Vacantis
}

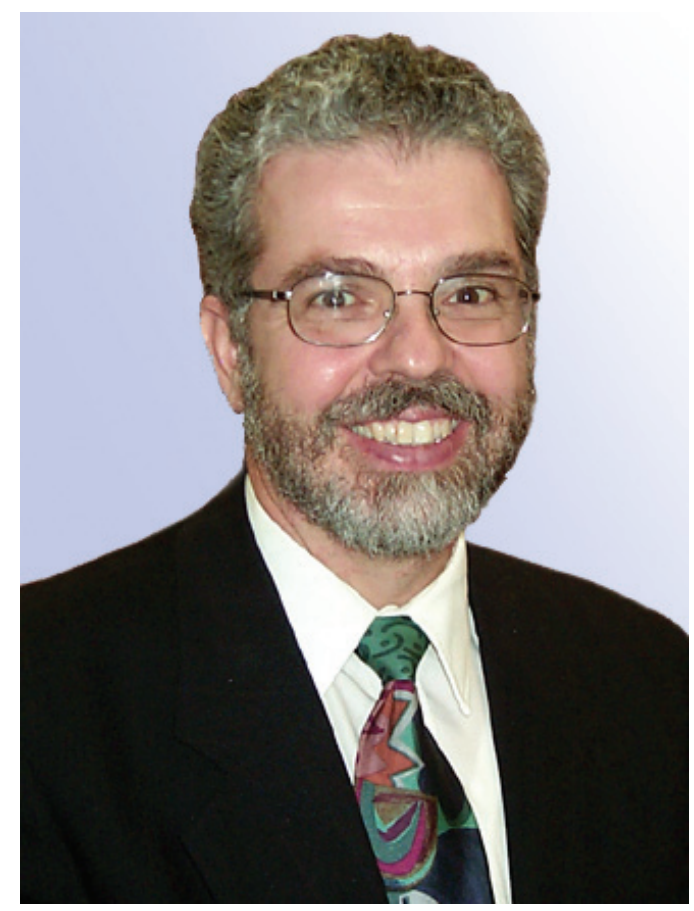

\section{Dr. Charles A. Vacanti, MD}

LeRoy VanDam/Benjamin Covino, Professor of Anaesthesia at Harvard Medical School

Anesthesiologist-in-Chief at the Brigham and Women's Hospital, Boston

Director of the laboratories for Tissue Engineering and Regenerative Medicine at the Brigham and Women's Hospital

President-Elect of the Society of Academic Anesthesiology Chairmen (SAAC) in the U.S.
There is no doubt that Dr. Joseph Vacanti and Dr. Charles Vacanti can be considered to be some of the "Stem cells of tissue engineering", as it has been put once by Dr. Peter Johnson, the former president of the Tissue Engineering Society International. They made some of the seminal observations that have enabled many people worldwide to develop many types of tissues.

A world-renowned leader in tissue engineering, Dr. Charles A. Vacanti is a co-founder of the Tissue Engineering Society, where he currently serves as emeritus governor, and is the co-editor of Tissue Engineering, the organization's official journal.

His research involves the generation of new functional tissue using a combination of living cells and synthetic biodegradable polymer scaffolds. He is especially interested in spinal cord regeneration.

Prior to his current position, Dr. Vacanti was chairman of the department of anesthesiology and a professor of anesthesiology and surgery at the University of Massachusetts Medical School, Worcester. He had also been on-staff at the UMass Memorial Medical Center in Worcester since 1994.

Dr. Vacanti has published more than 200 journal articles, book chapters and reviews, and abstracts. In addition to his research initiatives, he has also been granted approximately 20 patents in the field of tissue engineering and has more than a dozen pending.

$\mathrm{He}$ is a member of the American Society of Anesthesiologists, the International Anesthesia Research Society, Massachusetts Medical Society, Massachusetts Society of Anesthesiologists, and the Association of University Anesthesiologists.

It was the groundbreaking idea of Charles Vacanti together with others to visualize the idea behind tissue engineering to the public when he transplanted chondrocytes utilizing a polymercell construct to produce tissue-engineered cartilage in the shape of a human ear on the back of an immuno-compromised nude mouse, which was promptly dubbed Auriculosaurus. This disturbing image appeared in publications around the world and has ever since been perceived as the symbol of TE.

Dr. Joseph P. Vacanti as a surgeon-scientist in the field of pediatric surgery and transplantation surgery had realized that the major problem in transplantation was insufficient donor organs, and that this was a specific manifestation of the general problem of every field of reconstructive surgery, namely that of insufficient tissue. 


\title{
Tribute to the Vacantis
}

\author{
Dr. Joseph P. Vacanti, MD
}

John Homans Professor of Surgery, Harvard Medical School

Chief, Pediatric Surgery, Massachusetts General Hospital

Surgeon in Chief, MassGeneral Hospital for Children

Director, Laboratory of Tissue Engineering and Organ Fabrication

Director, Pediatric Transplantation

Co-Director, The Center for Regenerative Medicine

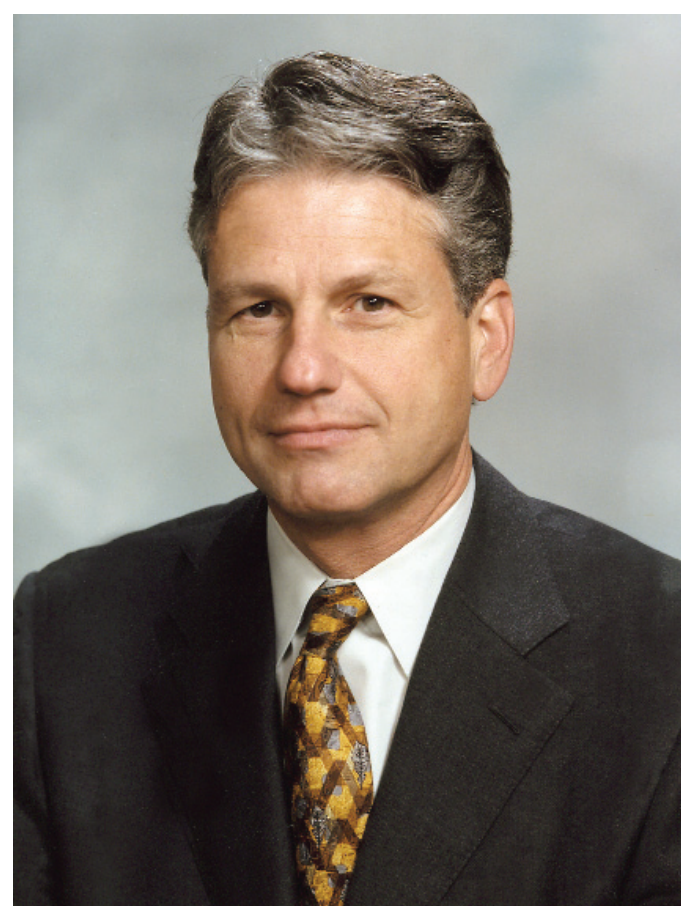

This insight was a stimulus for Dr. Vacanti to begin the conceptual development of the design of implantable systems which would act as devices to generate new tissue as permanent replacement structures. Working closely with Professor Langer at MIT, they developed an approach which used tissue-specific cells placed in devices composed of biodegradable, biocompatible polymers which, upon implantation, would signal vascular ingrowth while meeting the mass transfer diffusional requirements of the cells.

Dr. Joseph P. Vacanti received his MD degree from the University of Nebraska in 1974. $\mathrm{He}$ received his training in general surgery at the Massachusetts General Hospital from 1974 through 1981 and in pediatric surgery at The Children's Hospital, Boston from 1981 through 1983. He then received clinical training in transplantation from the University of Pittsburgh. He spent two years in the laboratories of Dr. M. Judah Folkman working in the field of angiogenesis from 1977 through 1979. Upon completion of his training, Dr. Vacanti joined the staff in surgery at Children's Hospital in Boston and began clinical programs in pediatric liver trans- plantation and extracorporeal membrane oxygenation. In the laboratory, he continued studies in biliary atresia and began work in the field of tissue engineering in 1985.

Dr. Vacanti is now John Homans Professor of Surgery at Harvard Medical School, Visiting Surgeon at Massachusetts General Hospital, Director of the Wellman 6 Surgical Laboratories, Director of the Laboratory of Tissue Engineering and Organ Fabrication and Director of Pediatric Transplantation at Massachusetts General Hospital, Boston.

He has authored more than 250 original reports, 44 review papers, 58 book chapters, and 419 abstracts. He has more than 73 patents or patents pending in the Canada, United States, Europe, and Japan.

Along the way, their brothers Frank and Marty Vacanti, who are also doctors and tissue engineering researchers, have joined the effort. In summary the Vacanti brothers have studied several different systems not only to demonstrate proof of principle in broad areas of surgery but also significantly contributed to uncover principles of design and biologic behavior. 
'Tissue Engineering' Review Series

EDITORIAL FOREWORD

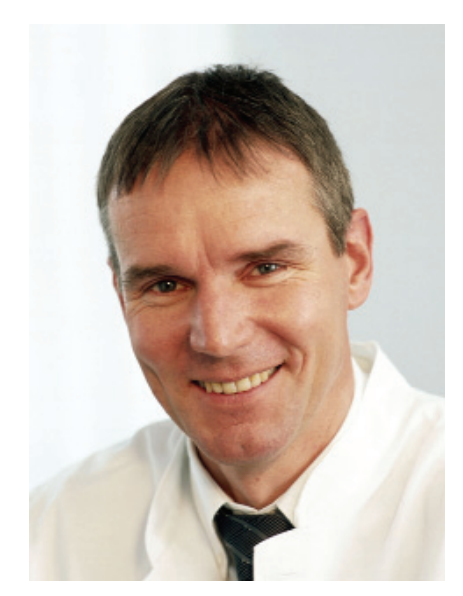

\title{
Future perspectives in tissue engineering
}

\author{
Raymund E. Horch * \\ Guest Editor of the "Tissue Engineering" Review Series
}

The term "Tissue engineering" had been defined at a National Science Foundation workshop as "the application of the principles and methods of engineering and life sciences toward the fundamental understanding of structure-function relationships in normal and pathological mammalian tissues and the development of biological substitutes that restore, maintain, or improve tissue function" [7].

Tissue engineering (TE) is a rapidly growing new interdisciplinary field of applied research that probably represents a prototype of future developments in science. Primarily such separate fields of science as engineering and life sciences have married to overcome traditional barriers in medical research. Tissue engineering crosses numerous medical and technical specialties. Cell biologists, molecular biologists, biomaterial engineers, microscopic imaging spe-

\footnotetext{
* Correspondence to: Raymund E. HORCH,

Department of Plastic and Hand Surgery, Laboratory of Tissue Engineering and Regeneration, (Director: R.E.Horch),
}

cialists, robotics engineers, computer-assisted designers, and developers of equipment such as bioreactors, where tissues are grown and nurtured, and finally legal advisors and marketing research specialists (product development, and medical implementation) are all involved in the practical process of tissue engineering. As a consequence permanently completely new disciplines seem to be created, such as the field of computer-aided tissue engineering (CATE), which encompasses computer-aided design (CAD), image processing, manufacturing and solid free-form fabrication (SFF) for modelling, designing, simulation and manufacturing of biological tissue and organ substitutes [10].

To put it more basically, TE combines engineering and biosciences together with clinical application - mainly in the various surgical specialties - in order to develop living substi-

University of Erlangen-Nuernberg, Krankenhausstrasse 12, D-91054 Erlangen, Germany, E.U.

E-mail: raymund.horch@chir.imed.uni-erlangen.de 
tutes for tissues and organs. This may allow the induction of true regeneration of tissue defects, and may well help to improve the treatment of many innate and aquired, especially chronic diseases and tissue defects by replacing or supporting the function of defective or injured body parts.

The key to its progress is an understanding between basic scientists, biochemical engineers, clinicians and industry. The main successful approaches to this mean so far have been progresses in the understanding of cell-cell interactions, the selection of appropriate matrices (cell-matrix interaction), and chemical signalling (growth factors).

Therefore the classical approach for engineering tissue has been the seeding of more or less biodegradable scaffolds with donor cells and/or growth factors, then culturing and implanting the scaffolds to induce and direct the growth of new, healthy tissue. So far, clinically successful applications include skin, cartilage and bone replacement. Intensive experimental research for reconstructive purposes addresses peripheral nerves, adipose tissue, muscle, urinary tubular structures, connective tissue (tendons, abdominal wall) cartilage, bone, and osteocartilagenous constructs (joints), heart valves and blood vessels, breast, small intestine, esophagus, pancreas, liver, and tracheal constructs $[1,5]$.

Until now in Asia and Europe governmental funding has included more basic research, whereas in the United States of America most funding has been for commercial development [8]. Future will show how or if this fact will influence the development of tissue engineering research.

Structural applications, such as the development of skeletal tissues, have become the fastest growing segment in tissue engineering research with stem-cell research programs spearheading the way [6]. The need for bone substitutes is particularly important. Bone substitutes are often required to help repair or replace damaged or diseased tissues in cases ranging from trauma, to congenital and degenerative diseases, to cancer, to cosmetics.

Currently available bone substitutes, including autografts, allografts, and synthetic materials, are the most implanted materials second only to transfused blood products worldwide. However, right now these substitutes are far from ideal and have many associated problems, e.g., autografting is expensive and can have significant donor site morbidity. On the other hand synthetic materials wear and do not behave like true bone. In this context the creation of alternative solutions by creating large-scale, tissue engineered bone with inherent vasculature is a very promising and definitely medically attractive aspect of latest research in TE. Using advanced CAD/CAM (computer-aided-design /computer-aided-manufacturing) techniques and appropriate bioreactor systems, capable of growing large-scale, customized bone substitutes that fit the individual patient's needs may be derived from CT or MRI data of the patient. Biodegradable scaffold structure (embedding donor cells and growth factors and possibly synthetic vasculature within the layers), cellular distributions, and growth factor concentrations of such synthetically grown bone could be spatially varied using selective deposition. Additional osteogenic cues, such as mechanical stimulus, could also be provided until the tissue was mature enough to be removed from the bioreactor and implanted into the patient.

Primary organ specific cells have been widely used for the generation of bioartificial tissues. Despite many advances efficient expansion of these cells remains challenging today. Stem cells - which by definition, are cells able to self-renew, with a single cell able to differentiate into multiple, functional cell types - on the other hand would theoretically overcome this limitiation. The stem cell field is also advancing rapidly, opening new options for therapy [2]. Stem cells also fulfill a second requirement: They can functionally reconstitute a given tissue in vivo. Mesenchymal stem cells for instance have been even used in clinical practice already. Pluripotent embryonic stem cells, which offer a potentially limitless source of cells for tissue engineering applications pose many new problems. The pluripotentiality of embryonic stem cells is also their limitation, and explains why they are not used clinically today [9].

The tissue engineering induced new interest into cell behaviour under culture conditions has led to the discovery of new cell types $[3,11]$. 
Since the blue-print for tissue differentiation is the genome it seems also reasonable to combine tissue engineering with gene therapy. This joined approach further augments the potential of both technologies. However the complexity of genetic manipulation of living cells prior to their implantation into artifical organs cannot be overestimated.

Although cells have been cultured, or grown, outside the body for many years, the possibility of growing complex, three-dimensional tissues - literally replicating the design and function of human tissue - is a more recent development. Bioreactors - dynamic cell culture systems that allow more control to generate larger volumes of cells when compared to conventional static-culture techniques - are only one possible way to further advance the field. The intricacies of this process require input from many types of scientists, including the problem solving expertise of engineers, hence the name tissue engineering.

Tissue engineering, however, must not be equated with the ex vivo construction of a living implant: presently the mammalian organism is unsurpassed as a bioreactor. If missing factors like cultured cells and an adaequate matrix are substituted, it may well occur that the complex threedimensional reconstitution of tissues will take place much better in vivo than in vitro. Mere biological matrices allow the attachment, proliferation, differentiation, migration and nutrition of various cell types in vivo but often lack the desired structural long term integrity [4]. On the other hand purely synthetic biomaterials so far have not achieved the quality of biological matrices, with regard to compatibility, architecture, binding sites and integration. Once again the combination of various scientific disciplines is necessary to further develop appropriate new materials or blending the properties of synthetic and biologic matrices in an ideal way.

Using tissue engineering technologies, it will hopefully one day be possible to truly regenerate or replace damaged tissues with laboratorygrown parts. With this issue a series of state of the art reviews featuring various disciplines of tissue engineering the Journal of Cellular and Molecular Medicine provides comprehensive information on the latest and most recent developments in this exciting new scientific field.

1. Bach AD, Beier JP, Stern-Staeter J, Horch RE. Skeletal muscle tissue engineering. J Cell Mol Med. 2004; 8: 413-22.

2. Caplan AI. Review: mesenchymal stem cells: cell-based reconstructive therapy in orthopedics. Tissue Eng. 2005; 11: 1198-211.

3. Hinescu ME, Popescu LM. Interstitial Cajal-like cells (ICLC) in human atrial myocardium. J Cell Mol Med. 2005; 9: 972-5.

4. Horch RE, Debus M, Wagner G, Stark GB. Cultured human keratinocytes on type I collagen membranes to reconstitute the epidermis. Tissue Eng. 2000; 6: 53-67.

5. Horch RE, Kopp J, Kneser U, Beier J, Bach AD. Tissue engineering of cultured skin substitutes. $J$ Cell Mol Med. 2005; 9: 592-608.

6. Knight MA, Evans GR. Tissue engineering: progress and challenges. Plast Reconstr Surg. 2004; 114: 26E-37E.

7. Langer R, Vacanti JP. Tissue engineering. Science 1993; 260: 920-6.

8. McIntire LV. World technology panel report on tissue engineering. Ann Biomed Eng. 2002; 30: 1216-20.

9. Radu E, Regalia T, Ceafalan L, Andrei F, Cretoiu D, Popescu LM. Cajal-type cells from human mammary gland stroma: phenotype characteristics in cell culture. $J$ Cell Mol Med. 2005; 9: 748-52.

10. Sun W, Darling A, Starly B, Nam J. Computer-aided tissue engineering: overview, scope and challenges. Biotechnol Appl Biochem. 2004; 39: 29-47.

11. Vacanti MP, Roy A, Cortiella J, Bonassar L, Vacanti CA. Identification and initial characterization of sporelike cells in adult mammals. $J$ Cell Biochem. 2001; 80: 455-60. 